Relations industrielles

Industrial Relations

\title{
Strikes and Lockouts in Canada, 1964. Economics and Research Branch, Department of Labor, Canada. 51 pages.
}

\section{Claude R. Têtu}

Volume 21, numéro 4, 1966

Congrès de l'ICRRI - 1966

1966 - CIRRI Annual Convention

URI : https://id.erudit.org/iderudit/027740ar

DOI : https://doi.org/10.7202/027740ar

Aller au sommaire du numéro

Éditeur(s)

Département des relations industrielles de l'Université Laval

ISSN

0034-379X (imprimé)

1703-8138 (numérique)

Découvrir la revue

Citer ce compte rendu

Têtu, C. R. (1966). Compte rendu de [Strikes and Lockouts in Canada, 1964. Economics and Research Branch, Department of Labor, Canada. 51 pages.]

Relations industrielles / Industrial Relations, 21(4), 657-657.

https://doi.org/10.7202/027740ar

Tous droits réservés @ Département des relations industrielles de l'Université Laval, 1966
Ce document est protégé par la loi sur le droit d'auteur. L'utilisation des services d'Érudit (y compris la reproduction) est assujettie à sa politique d'utilisation que vous pouvez consulter en ligne.

https://apropos.erudit.org/fr/usagers/politique-dutilisation/ 
Strikes and Lockouts in Canada, 1964. Economics and Research Branch, Department of Labor, Canado. 51 pages.

Le fascicule que nous vous présentons, couvre tous les arrêts de travail dont a pris connaissance le Ministère du Travail du Canada. Parmi les considérations générales du début, il est intéressant de noter que plus de la moitié du temps de travail perdu durant l'année est attribuable à quatre grandes grèves. Ce furent celles à la General Motors, à la Presse, chez Macmillan Bloedell and Powell River et enfin au Star, Telegram et Globe and Mail de Toronto.

Cette courte publication se subdivise en sept graphiques et dix tableaux. La plupart du temps on y mesure la durée des arrêts de travail en a homme-jour ». On obtient cette mesure en multipliant le nombre de journées de travail perdues par le nombre de travailleurs impliqués. II est évident que cette compilation statistique nous fournit des renseignements précieux sur les arrêts de travail au Canada. Cependant une étude même attentive du document laisse un certain nombre de questions sans réponse. Par exemple, dans le calcul du temps perdu, on ne considère que les travailleurs directement impliqués dans l'arrêt de travail. En aucun cas on n'a essayé d'évaluer les répercussions d'un arrêt de travail sur les autres travailleurs de l'industrie, de la région économique ou de I'usine impliquée.

Une seconde remarque concerne l'absence d'une distribution des arrêts de travail par province ou par secteurs économiques. La seule distribution qui nous est fournie est basée sur les secteurs industriels. De cette façon nous avons une vue d'ensemble de la situation canadienne qui est fort intéressante mais qui jusqu'ò un certain point ne nous satisfait pas.

En concluont, il convient de signaler que ce périodique est publié chaque année dans les deux langues. Facilement accessible, il peut devenir un instrument d'information ou de recherche très valable.

\section{Claude R. Têtu}

The Global Businessman, edited by Charles F. Stewort, Holt, Rinehart and Winston of Canada Ltd., Toronto, 1966, 264 pages.

Cet ouvrage pose comme première donnée que le marché américain des affaires est déficient pour un grand nombre d'industries américaines expansionnistes. Par conséquent i) faut que les hommes d'affaires aient une vue de plus en plus globale du déroulement de leurs opérations. Cette vision des affaires $n^{\prime}$ est pas, cependant, sans causer de graves problèmes à ces gens-là car ils doivent surmonter des obstacles tels que les différences dans le langage, la culture, les idéologies politiques et économiques.

Cet intéressant volume est divisé en trois parties principales. La première traite de l'événement et inclut certains focteurs qui affectent d'une façon vitale les opérations d'une industrie mais sur lesquels la direction n'exerce peu ou pas de contrôle. Parmi ces questions problématiques, on dénombre particulièrement des difficultés de croissance économique et de stabilité de la monnaie ainsi que de la balance des paiements. Un autre facteur qu'il ne faut pas négliger non plus: les politiques étrangères de commerce qui font appel à ce dont nous avons parlé plus haut: la diversité des idéologies politiques et économiques. Qu'il suffise de penser, à cette fin, à I'Union Soviétique et nous aurons alors à l'idée toute la précocité des problèmes à résoudre.

La deuxième partie de ce volume traite de la façon dont se comportent les différentes compagnies dans le contexte global du monde des offaires. A ce sujet, on a étudié l'activité de deux compagnies étrangères dont l'attitude peut se révéler pertinente quant la compétition sur le marché mondial du travail et également quant aux exemples que l'entreprise américaine peut en tirer.

Enfin, la troisième et dernière partie de l'ouvrage est centrée sur une étude de cas. On constate alors, avec grand intérêt, que l'industrie en général et spécialement celle du pétrole doit affronter des problèmes de contrôle, d'instabilité momentanée et de fluctuations des marchés. Cette expérience montre donc, en dernière analyse, de quelle manière l'industrie pétrolière a réussi avec beaucoup de succès à s'implanter sur le marché mondial et à y occuper la place enviable qu'on lui connait.

En terminant, on peut dire que l'étude qui nous est présentée revêt des caractères à la fois pratiques et logiques, ce qui fait qu'elle rencontre l'intérêt de l'homme d'affaires qui s'identifie aux problèmes épineux de tous les jours. 